\title{
Percepción de los docentes de primaria en ejercicio, acerca de las matemáticas y su enseñanza en relación con los programas oficiales del MEP
}

\author{
Perceptions of In-Service Elementary School Teachers towards Mathematics and its \\ Teaching Regarding the Official Programs of the Ministry of Public Education
}

\author{
Edwin Chaves-Esquivel \\ edwin.chaves.esquivel@una.cr \\ Universidad Nacional \\ Heredia, Costa Rica \\ Marianela Alpízar-Vargas \\ malpiza@una.cr \\ Universidad Nacional \\ Heredia, Costa Rica \\ Ana-Lucía Alfaro-Arce \\ aalfar@una.cr \\ Universidad Nacional \\ Heredia, Costa Rica
}

Recibido-Received: 2/mar/2015 / Aceptado-Accepted: 27/may/2015 / Publicado-Published: 31/ene /2016.

\begin{abstract}
Resumen
El presente estudio se ha realizado en el marco del proyecto de investigación La utopía en la enseñanza de las matemáticas en primaria, que se desarrolla en la Escuela de Matemáticas de la Universidad Nacional de Costa Rica. En este artículo se reporta la percepción que un grupo de docentes de primaria en ejercicio tiene sobre las matemáticas y su enseñanza. La información se recabó por medio de un cuestionario de 66 preguntas, dirigidas a determinar su percepción de las matemáticas y su enseñanza en relación con los actuales programas del Ministerio de Educación Pública; además, de su percepción sobre la formación inicial recibida y en los procesos de capacitación. Se obtuvo la respuesta de 87 docentes en servicio. Dentro de los principales hallazgos están: su percepción fue positiva en cuanto a las matemáticas y su enseñanza; el uso de la resolución de problemas y las aplicaciones de las matemáticas en la clase, en vez de procedimientos rutinarios y reglas memorizadas, han tomado relevancia; sin embargo, este aspecto se debe reforzar y profundizar en los procesos de capacitación que se ofrecen. Aunque creen que son importantes las creencias y la percepción de sus estudiantes al momento de plantear actividades de clase, un número importante de docentes opina que las matemáticas pueden provocar inseguridad en el alumnado. Sienten poca satisfacción en lo relativo a aspectos teóricos, estrategias de enseñanza y de evaluación; y piensan que su formación inicial no guarda estrecha relación con la labor profesional que realizan en las aulas.
\end{abstract}

Palabras claves: Educación primaria;, educación matemática, percepciones, programas de estudio. 
UNICIENCIA Vol. 30, No. 1, pp. 31-55. Enero-Junio, 2016.

ISSN Electrónico: 2215-3470

URL: www.revistas.una.ac.cr/uniciencia

DOI: http://dx.doi.org/10.15359/ru.30-1.3

Email: revistauniciencia@una.cr

\begin{abstract}
This study was carried out as part of the research project Utopia in Teaching Mathematics in Elementary School, executed by the School of Mathematics at National University of Costa Rica. This article shows the perception of a group of elementary school educators about mathematics and its teaching. The information was gathered through a 66 question questionnaire, designed to assess the perception of elementary school teachers about mathematics and its teaching in relation to the current programs provided by the Ministry of Public Education, and also their perception of the initial training they obtained when they were studying and in processes of training. Among the main findings are: beliefs of teachers tend to be positive about mathematics and its teaching, and educators teaching primary school levels tend to use more problem-solving exercises in which students can apply their mathematics knowledge, rather than having learners work on routine exercises and memorize rules. However, this aspect should be strengthened and deepened in the processes of training being offered by educational authorities. Although participants considered that students' beliefs and perceptions are quite important when framing classroom activities, a significant number of them pointed out that mathematics can make students feel insecure. Teachers feel little satisfaction regarding the theoretical aspects, teaching strategies and assessment of mathematics in public schools; they stated that their initial training is not concomitant with the work they must carry out in elementary school classrooms.
\end{abstract}

Keywords: primary school education; Mathematics education; beliefs; perceptions; study programs.

Tradicionalmente, los aspectos emocionales han sido dejados de lado en el proceso de enseñanza y aprendizaje. Sin embargo, diferentes estudios han demostrado que dichos aspectos constituyen un pilar fundamental en el ámbito educativo. Esto se presenta en mayor medida en el área de matemáticas, donde normalmente se ha presentado una serie de prejuicios en contra de la disciplina, los cuales provocan que estudiantes y docentes se sientan emocionalmente afectados al momento de abordar los procesos de enseñanza, aprendizaje y evaluación de la disciplina (Chaves, Castillo y Alpízar, 2009; Chaves, Castillo y Gamboa, 2008; Estrada, 2002; McLeod y McLeod, 2002).

$\mathrm{Al}$ analizar la propuesta plasmada en los actuales programas de matemáticas para primaria y secundaria, se establece:

En el aprendizaje son decisivas la motivación y el interés y en general todas las dimensiones afectivas, por lo que se adopta aquí una visión integral y humanista sobre la enseñanza y aprendizaje de las Matemáticas. No se pueden generar actitudes y creencias positivas hacia la enseñanza y aprendizaje de las Matemáticas sin que los programas las incorporen de forma explícita y ofrezcan medios pedagógicos en esa dirección. (MEP, 2012, p. 45)

Indican, además:

Las actitudes están estrechamente ligadas a las creencias, que incluso se toman de los ámbitos familiares y culturales de la sociedad. Conocer estas creencias y revertirlas hacia otras de mayor positividad hacia las Matemáticas debe ser un propósito a incorporar en todos los niveles educativos. Identificar y transformar las percepciones negativas en positivas debe ser parte de los fines de una educación anclada en los requerimientos de la sociedad en que vivimos. (MEP, 2012, p. 45)

En este sentido, la percepción que el personal docente tenga sobre las matemáticas y su enseñanza cobra especial interés para que se alcance una implementación exitosa delos programas 
de matemáticas del MEP. Por esta razón, en el presente artículo, se pretende determinar dicha percepción en un grupo de educadores de primaria en ejercicio.

Pozo y Scheuer (2006) establecen que, dentro de la dinámica educativa moderna, es de gran interés el identificar las creencias del profesorado como una de las variables que mayoritariamente inciden en la práctica educativa, por lo que se debe conocer y reflexionar sobre estas creencias y percepciones, de modo que se pueda valorar la forma en que ellas pueden generar una conducta cognitiva determinada en su labor de aula. Es decir, el sistema personal de creencias de cada docente sobre las matemáticas y su enseñanza influye directamente sobre la labor de aula. Por esta razón, en el caso de percepciones negativas, se requieren estrategias que reviertan el problema de modo que sea posible mantener congruencia con la propuesta educativa actual.

En el estudio realizado por Bosch y Gascón (2001), se analizaron algunas propuestas vinculadas con prácticas docentes en el aula de matemáticas. Se concluye que estas propuestas se fundamentan en una interacción afectiva entre docente y estudiantes, que debe ser tomada en cuenta al momento de planificar la acción de aula.

Específicamente, con base en el rol docente, Bosch y Gascón (2001) explican su comportamiento en el aula con base en:

a) El conocimiento del personal docente: en contenidos matemáticos, en aspectos pedagógicos generales y en mecanismos sobre didáctica específica de las matemáticas.

b) Las creencias del profesorado: sobre las matemáticas y sobre el aprendizaje de las matemáticas.

c) Las actitudes del profesorado.

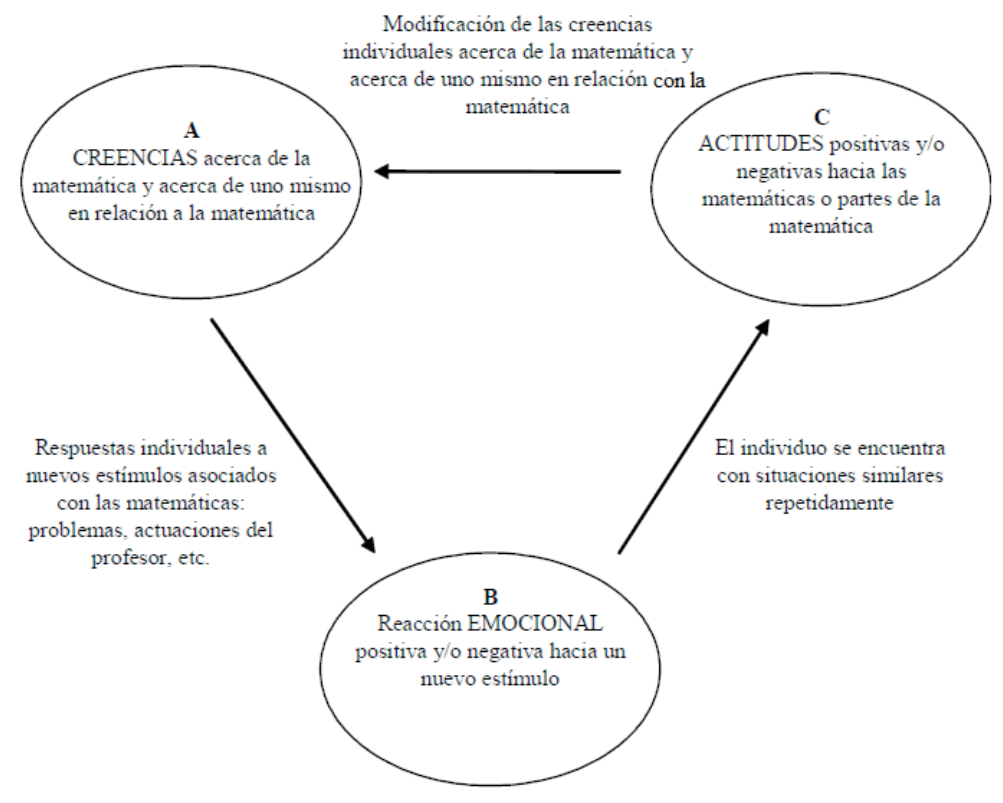

Figura 1. Actitudes y conocimientos elementales en la formación del profesorado. Estrada (2002, p. 52). Adaptación. 
Para Bosch y Gascón (2001), con este modelo se observa que el aprendizaje estudiantil está explicado por una serie de variables; se evidencia que no basta con tener docentes con buena preparación tanto en conocimientos matemáticos, como pedagógicos y didácticos; pues las percepciones tienen igualmente una trascendencia vital para generar dicho aprendizaje.

Al respecto, Flores (1998) señala quela formación integral de docentes es un área fundamental de interés en la educación matemática, esto debido a que la labor docente es relevante en la enseñanza de las matemáticas. Flores (1998, p. 1) señala:

Esta importancia se acrecienta en períodos de reforma educativa, ya que difícilmente se podrá aplicar dicha reforma si los profesores, como principales agentes que tienen que ponerla en práctica, no la sienten como necesaria, no la asumen como propia y no aportan los esfuerzos necesarios para realizarla.

Las investigaciones anteriores son muy pertinentes para la realidad costarricense, debido a que, a partir del año 2013, se han puesto en práctica nuevos programas educativos de matemáticas en primaria y secundaria de la educación pública, los cuales van a estar en un proceso de transición hasta el año 2016. Estos programas plantean una transformación radical tanto en los fundamentos teóricos que lo sustentan, como en los demás componentes del currículo. Su objetivo principal consiste en la búsqueda del fortalecimiento de las capacidades cognoscitivas para enfrentar los retos de la sociedad moderna, en la cual el conocimiento, la información, y la demanda de habilidades y capacidades de razonamiento lógico y de toma de decisiones basadas en evidencia concreta tienen especial relevancia. Para el logro de dicho objetivo, se plantean dos propósitos básicos: en primer lugar cada estudiante debe asumir la responsabilidad de participar activamente en la construcción de su propio aprendizaje y, en segundo lugar, la acción docente debe enfocarse en la creación y planteamiento de suficientes situaciones de aprendizaje que faciliten el logro del primer propósito en forma motivadora (MEP, 2012).

Desde esta óptica, se requiere que el personal docente se sienta identificado con esta propuesta, que tenga una clara concepción sobre la importancia de las matemáticas para la formación ciudadana, debido a su potencial para comprender el entorno, y que sea capaz de reflexionar sobre su propio quehacer, con el propósito de repensarlo y proponer estrategias de mejora.

Flores (1998) indica que parte del oficio docente consiste en reflexionar sobre sus propias prácticas, hecho que debe iniciar durante la formación, en donde se debería reflexionar sobre las concepciones vinculadas con el conocimiento matemático, y su futuro papel como educador o educadora de la disciplina.

De acuerdo con lo anterior, el estudio de las percepciones del personal docente sobre las matemáticas y su enseñanza, es de sumo interés en el momento actual; más aún si se concentra el estudio en docentes de primaria, por su importancia en la formación integral de la ciudadanía.

\section{Marco teórico}

\section{Estrategia didáctica para la enseñanza de las matemáticas según el MEP}

En los programas de estudio de matemáticas del Ministerio de Educación Pública (MEP) se promueve una acción de aula centrada en la resolución de problemas vinculados con contextos reales, físicos, sociales y culturales. Por medio de esta estrategia se procura que cada estudiante se 
ISSN Electrónico: 2215-3470

DOI: http://dx.doi.org/10.15359/ru.30-1.3
UNICIENCIA Vol. 30, No. 1, pp. 31-55. Enero-Junio, 2016.

URL: www.revistas.una.ac.cr/uniciencia Email: revistauniciencia@una.cr

identifique con el entorno y con el uso de las matemáticas como herramienta para comprender y modelar las situaciones de la cotidianidad estudiantil. En este sentido, la mediación pedagógica adopta premisas fundamentales constructivistas, tal como lo establece la política educativa vigente para el país, la cual promueve construcción activa de los aprendizajes por parte de cada estudiante. (MEP, 2012). Con este enfoque, se promueve el desarrollo de habilidades vinculadas directamente a las áreas matemáticas.

El aprendizaje de los conceptos o conocimientos disciplinares por medio de la resolución de problemas debe ser concentrado en cuatro pasos básicos: "propuesta de un problema, trabajo estudiantil independiente, discusión interactiva y comunicativa y clausura o cierre" (MEP, 2012, p. 14).

El desarrollo de un problema por parte de los grupos de estudiantes puede ocupar una o más lecciones. Esta propuesta se contrapone con la enseñanza tradicional que ha estado centrada en el desarrollo de los tópicos matemáticos en abstracto por parte del personal docente, con el planteo de ejemplos, prácticas rutinarias y mecánicas.

Por lo anterior, el planteo y resolución de problemas constituye una constante durante todo el proceso educativo, lo que incluye "el reforzamiento, movilización y aplicación de los conocimientos aprendidos" (MEP, 2012, p. 15).

Aunque se promueve el empleo de problemas vinculados con contextos reales, la implementación de problemas abstractos para situaciones particulares constituye también una importante herramienta del proceso educativo. Con esto, el MEP pretende "la construcción de capacidades para la manipulación de los objetos matemáticos cuya naturaleza es abstracta. La estrategia asumida se propone fundamentar pedagógicamente el paso desde lo concreto a lo abstracto" (MEP, 2012, p. 15).

\section{La potenciación de actitudes y creencias en torno a las matemáticas}

En los programas de estudio del MEP, se promueve potenciar actitudes y creencias positivas en torno a las matemáticas, de modo que este tema se incluye como uno de los cinco ejes disciplinares. Se vinculan las actitudes de estudiantes con las creencias con que ingresan al ámbito educativo, fruto de su relación con el entorno familiar y comunal. En este sentido, se plantea que el personal docente debe reconocer estas creencias y debe redirigirlas hacia la consecución de actitudes que le ayuden a comprender dicho entorno de una manera objetiva.

Las actitudes que se desea promover son:

Perseverancia. Se promueve la identificación que con trabajo, dedicación y persistencia es posible resolver problemas matemáticos de gran complejidad.

Confianza en la utilidad de las matemáticas. Las actividades educativas que se planteen deben dejar en evidencia la utilidad de las matemáticas para lograr una menor comprensión del entorno.

Participación activa y colaborativa. La acción de aula debe permitir al estudiantado involucrarse directamente en la generación del conocimiento nuevo, en colaboración con sus compañeros y compañeras.

Autoestima en relación con el dominio de las matemáticas. Para lograr esta autoestima, se propone al personal docente el diseño de problemas que, en forma escalonada, planteen diferentes niveles de profundidad que permitan al estudiantado ir profundizando, paulatinamente, en el logro de habilidades matemáticas, de modo que conforme vaya avanzando adquiera la confianza necesaria para pasar a la siguiente etapa. 
UNICIENCIA Vol. 30, No. 1, pp. 31-55. Enero-Junio, 2016.

ISSN Electrónico: 2215-3470

URL: www.revistas.una.ac.cr/uniciencia

Email: revistauniciencia@una.cr

Respeto, aprecio y disfrute de las matemáticas. Aunque, durante sus vidas, no todas las personas van a relacionarse con las matemáticas de igual forma, y posiblemente van a requerir las mismas habilidades matemáticas, resulta fundamental que unas y otras valoren las matemáticas y disfruten de su uso dentro de las actividades que realicen.

$\mathrm{Al}$ ser un eje disciplinar, estos elementos deben estar presentes en forma transversal en todos los años del proceso educativo (MEP, 2012).

\section{Las áreas matemáticas según los programas de estudio}

Desde la perspectiva emotiva, se indica que en el primer ciclo, el personal docente debe crear las condiciones para generar una percepción inicial positiva de sus estudiantes hacia la disciplina, sobre una base afectiva para profundizar sus conceptos y procedimientos en el siguiente ciclo.

En este sentido, en el primer ciclo interesa, fundamentalmente, identificar ciertas ideas y creencias con que el estudiantado llega a la escuela, con el propósito de que sirvan de referencia para la adquisición de ciertas habilidades vinculadas con el logro de conocimientos intuitivos tales como la ubicación espacial, comparación de tamaños, longitudes, espesores y cantidades de un modo cualitativo (mucho, poco, entre otros). De esta manera, se incursiona en las áreas de geometría, medidas, y estadística y probabilidad que tienen un peso relativo similar en cuanto a contenidos y habilidades por desarrollar, mientras que el área de números constituye la mayor parte, y relaciones y álgebra aparecen con una extensión menor (MEP, 2012).

Desde este punto de vista, en el área de números, las actividades que desarrolle el personal docente deben conducir a afianzar conocimientos, procesos matemáticos y actitudes positivas siempre alrededor de las representaciones numéricas y otros aspectos de esta misma área. Desde el inicio, se propone realizar una conexión entre el concepto intuitivo de número con su sentido numérico, que corresponde a la posición absoluta y relativa de los números, donde lo relevante es potenciar la estimación y el cálculo mental, pues con ello se logran procesos cognitivos vitales para el aprendizaje de las matemáticas. Aunque la memorización de procedimientos, actividad propia de la enseñanza tradicional, no se promueve en los nuevos programas, existen algunos procedimientos que deben ser memorizados (algoritmos de operaciones básicas: suma, resta y multiplicación), debido a que son herramientas de vital importancia para la adquisición de otras habilidades en diferentes áreas (MEP, 2012).

Por su parte, en relaciones y álgebra se pretende generar habilidades para la comprensión de patrones y relaciones, así como para iniciar la manipulación (leer y escribir) de símbolos básicos de las operaciones matemáticas y la representación en la recta numérica. En cuanto al área de medidas se plantea una estrecha relación con números, pero en una dimensión diferente debido a que la acción de medir se diferencia con la de resolver operaciones y hacer cálculos; pero se utiliza el sentido numérico. Algo similar ocurre en estadística y probabilidad, la cual utiliza números al introducir la noción de dato, que involucra información cualitativa y cuantitativa. En esta área, al finalizar el primer ciclo, se propone que el estudiantado haya adquirido habilidades para emplear estrategias de recolección, resumen, presentación y análisis de información. Asimismo, debe identificar entre actividades aleatorias y no aleatorias, e identificar eventos más o menos probables. Por su parte, en geometría se propone generar habilidades hacia la visualización (ubicación, reconocimiento de formas geométricas en el contexto), la manipulación y descripción de figuras geométricas, y algunas relaciones entre ellas, uso de vocabulario geométrico básico, resaltan el uso de manipulaciones y el trazado de figuras. 
ISSN Electrónico: 2215-3470

DOI: http://dx.doi.org/10.15359/ru.30-1.3
UNICIENCIA Vol. 30, No. 1, pp. 31-55. Enero-Junio, 2016.

URL: www.revistas.una.ac.cr/uniciencia Email: revistauniciencia@una.cr

Para el segundo ciclo, se indica en los programas que:

Las Matemáticas se colocan en una doble perspectiva: por un lado, afirman y amplían los conceptos y procedimientos fundamentales que se han aprendido en el Primer ciclo; por otro lado, hacia el final del ciclo se introducen conceptos y habilidades que conectan con la educación Secundaria, con características distintas a las que predominan en la educación Primaria. (MEP, 2012, p. 184)

Se plantea que ante la incorporación de conocimientos cognitivamente más complejos en todas las áreas, se requiere potenciar las actitudes positivas, que favorezcan la adquisición de las nuevas habilidades.

Nuevamente el área de números continúa teniendo un mayor peso relativo en cuanto a la cantidad de contenidos y habilidades por desarrollar, mientras que las áreas de medidas, geometría, relaciones y álgebra y estadística y probabilidad ocupan una ponderación similar en cuanto a cantidad de contenidos y habilidades. En números se amplía el análisis a números cada vez más grandes y al trabajo con decimales, fracciones y representaciones. Además se introduce el algoritmo de la división. En relaciones y álgebra se continúa con un trabajo similar al desarrollado en el primer ciclo, es decir, se trabaja con relaciones y patrones numéricos; pero ahora incluye la representación de puntos en un plano cartesiano y relaciones entre cantidades que varían, además del razonamiento proporcional que va a ser de mucha utilidad en la secundaria. En estadística y probabilidad se profundizan los conceptos y habilidades del primer ciclo, incorporando nuevas técnicas estadísticas, ampliando el análisis hacia la determinación de probabilidades con base en la definición clásica o laplaciana. En medidas se introduce el sistema métrico decimal, se ofrece un mayor énfasis a la estimación de mediciones en diferentes contextos. Finalmente, en geometría se continúa con el análisis de la ubicación espacial y con la visualización de formas geométricas tanto en el plano como en el espacio, además, se profundiza también en el estudio de propiedades de los elementos geométricos (MEP, 2012).

En general, el currículo actual no subestima el papel de los contenidos matemáticos, sino que por medio de los conocimientos matemáticos se organiza el plan de estudios en cada ciclo y año lectivo y las habilidades, que se espera sean aprendidas, en torno a ellos. Sin embargo, a pesar de que los conocimientos matemáticos constituyen la base de los programas, los contenidos teóricos no son el centro del proceso educativo; se procura favorecer el desarrollo de habilidades en el estudiantado para que pueda enfrentar los retos a los que está expuesto en la sociedad moderna.

Para conseguir los objetivos propuestos en los programas de estudio, la mediación pedagógica se convierte en uno de los pilares fundamentales. Esta mediación debe llevarse a cabo mediante diferentes estrategias didácticas. Con ellas se propone realizar cinco procesos básicos: "razonar y argumentar; plantear y resolver problemas; conectar, establecer relaciones; representar de diversas formas (gráficas, numéricas, simbólicas, tabulares, etc.) y comunicar, expresar ideas matemáticas formal y verbalmente"(MEP, 2012, p. 11)

Con el fortalecimiento de estos procesos se pretende que el estudiantado pueda adquirir las habilidades necesarias para realizar operaciones y procesos matemáticos de una complejidad gradual en la resolución de los problemas que se le plantean. Se pretende potenciar el rigor y la capacidad matemática para determinar las soluciones a problemas que buscan modelar situaciones de la realidad. Al respecto se indica: 
UNICIENCIA Vol. 30, No. 1, pp. 31-55. Enero-Junio, 2016.

ISSN Electrónico: 2215-3470

URL: www.revistas.una.ac.cr/uniciencia

Email: revistauniciencia@una.cr

Para la aplicación, matematización o modelización de diversas situaciones, así como de lograr mayores niveles analíticos en la justificación y argumentación matemática. Esto no se logra por medio de la "amplitud", abarrotando los programas de contenidos, sino seleccionando bien cuáles son los contenidos necesarios para lograr "rigor y profundidad" en el manejo de los procesos y el lenguaje matemático. Para lograr esto, los nuevos programas utilizan cinco ejes:

- La resolución de problemas como estrategia metodológica principal.

- La contextualización activa como un componente pedagógico especial.

- El uso inteligente de tecnologías digitales.

- La potenciación de actitudes y creencias positivas en torno a las Matemáticas.

- El uso de la Historia de las Matemáticas. (MEP, 2012, p. 11)

Los aspectos anteriores constituyen una parte esencial de los fundamentos teóricos que sustentan los programas de estudio de matemáticas, por lo que deben ser tomados en cuenta al momento de analizar la forma en que docentes de primaria conciben estos elementos en su articulación con el trabajo que realizan en el aula.

\section{Formación de docentes de primaria}

Como se ha venido señalando, el personal docente constituye una pieza medular en el proceso de enseñanza y aprendizaje, más aun en el caso de la educación primaria, donde el alumnado está en la etapa de la niñez; ya que debe favorecer un proceso de enseñanza de la asignatura correspondiente, pero además, orientar su desarrollo personal y social. Esto quiere decir que los educadores y educadoras deben prepararse en muchas áreas para poder afrontar su labor exitosamente.

En Costa Rica, la formación inicial docente es una tarea encargada a las universidades públicas y privadas que ofertan programas diversos, con mallas curriculares diferentes. En esta variabilidad existente hay pocas que dan cuenta de la calidad que ofrecen. Aunque uno de los mecanismos para mejorar la calidad de las carreras son los procesos de acreditación por parte del Sistema Nacional de Acreditación de la Educación Superior (SINAES), en los planes curriculares revisados en el estudio realizado por Alfaro, Alpízar, Morales, Ramírez y Salas en 2013, se encontró que la única universidad que tiene una carrera relacionada con I y II Ciclos de la Educación General Básica acreditada fue la Universidad Nacional. Este estudio analizó el plan de estudios de 15 universidades privadas y tres estatales que ofrecían, en el momento de la investigación, una carrera relacionada con la enseñanza para I y II Ciclos de la Educación General Básica.

En Costa Rica, el personal docente encargado de I y II Ciclos de la Educación General Básica debe impartir varias asignaturas a un mismo grupo de estudiantes. Generalmente, en el I Ciclo imparten las cuatro asignaturas básicas: matemáticas, español, ciencias y estudios sociales y en el II Ciclo, en algunas instituciones se imparten solamente dos de ellas, si se tiene el personal suficiente. Cabe destacar que, en algunas zonas rurales, el modelo de escuela es unidocente, por lo que un solo docente imparte todas las asignaturas y a todos los niveles.

Para Alfaro et al. (2013), el personal docente de primaria debe tener una formación inicial integral que no solo incluya conocimientos de pedagogía y didáctica general sino que, además, contemplen un conocimiento básico de las disciplinas específicas que deben enseñar y de los aspectos didácticos relacionados con cada una de ellas. 
Según Barrantes, Bolaños, Céspedes, Delgado, Freer, Padilla y Víquez, (2010), en el año 2009, veinticinco universidades tenían carreras profesionales relacionadas con Educación Básica en I y II Ciclos (información tomada de la página Web del Consejo Nacional de Enseñanza Superior Universitaria Privada y de la oferta de carreras de las universidades estatales). Sin embargo, para el 2012, en algunos de estos centros de educación dicha carrera estaba inactiva y ninguna de las activas tenía un énfasis en matemática (Alfaro et al., 2013).

En cada uno de los planes de formación para docente de primaria se tratan las asignaturas básicas: español, ciencias estudios sociales y matemáticas; algunas materias de psicología, currículo, desarrollo de la niñez y didácticas generales para ese nivel educativo.

Según el Cuarto Informe Estado de la Educación (Programa Estado de la Nación, 2013):

Actualmente la oferta conjunta de carreras en el área de Educación asciende a 259 opciones, de las cuales solo el 5,8\% está acreditado. Entre 2010 y 2011 se graduaron 21.446 docentes, que representan más de una quinta parte del total de graduados del país. La mayoría procedían de universidades privadas. (p. 165)

A pesar de esa amplia gama de opciones, Alfaro et al. (2013) consideran que las universidades formadoras de docentes de primaria no producen profesionales con la preparación suficiente y necesaria en matemáticas para lograr que se desenvuelvan de la mejor manera en esta asignatura. En la mayoría de los casos, para su bachillerato universitario solo llevan dos cursos de matemáticas que contienen, en algunos, los contenidos básicos de dicha asignatura y su didáctica (Alpízar, Barrantes, Bolaños, Céspedes, Delgado, Freer, Padilla y Víquez, 2012; Alfaro et al., 2013). Además, si el énfasis de su programa de estudios no es de matemática, sino bilingüe o en letras, el abordaje para las matemáticas es, generalmente, menor.

Es evidente que solamente con dos cursos de matemática no es posible que adquieran los conceptos teóricos de la asignatura y que, además, tengan clara la manera en que deben desarrollarse esos contenidos para favorecer el proceso de enseñanza y aprendizaje en sus estudiantes, en concordancia con el perfil docente que requieren los programas de matemáticas del MEP.

Tal como se ha citado, dichos programas demandan de docentes con muy buena preparación, que posean dominio en los temas que van a enseñar y sean capaces de contextualizarlos a la realidad, además de aplicar estrategias adecuadas para su enseñanza.

En este sentido, cobra vital importancia el desarrollo profesional que el personal docente pueda lograr mediante capacitaciones efectivas que le permitan adquirir habilidades necesarias para el abordaje de las matemáticas en el aula.

[En Costa Rica] el desarrollo profesional de los docentes en servicio está a cargo del MEP, que cuenta para ello con el Instituto de Desarrollo Profesional Uladislao Gámez Solano (IDP-UGS) y con el llamado "Plan 200", un mecanismo creado en 2006 para aprovechar los doscientos días del curso lectivo y que tiene entre sus objetivos realizar actividades de capacitación y desarrollo profesional durante dos semanas al final del año. (Programa Estado de la Nación, 2013, pp. 165-166)

Aunque se han logrado algunos avances y la capacitación ofrecida a docentes de primaria sobre las matemáticas y su enseñanza, del 2011 a la fecha, ha girado alrededor de los 
contenidos curriculares de los nuevos Programas de estudio de Matemáticas, aún falta mucho por hacer. Como lo señaló el Cuarto Informe del Estado de la Educación (Programa Estado de la Nación, 2013, p. 166):

No se logró aprobar una política nacional de desarrollo profesional que oriente la labor del IDP-UGS. Entre 2010 y 2012 los esfuerzos del Instituto se concentraron en brindar capacitación sobre los nuevos programas de estudio aprobados en distintas asignaturas. El "Plan 200", que está a cargo de la Dirección de Desarrollo Curricular del MEP con apoyo de las universidades y la Fundación Omar Dengo, siguió ejecutándose, pero sin mayor evaluación por parte del MEP, pese a que existe una serie de aspectos que requieren revisión.

\section{Metodología}

El presente estudio se ha realizado en el marco del proyecto de investigación denominado: $\mathrm{La}$ utopía en la enseñanza de las matemáticas en primaria, que se desarrolla en la Escuela de Matemáticas de la Universidad Nacional de Costa Rica. Dicho proyecto tiene como objetivo general determinar estrategias didácticas que pueden desarrollarse para contribuir en el cambio que se debe propiciar en el ámbito nacional en la enseñanza de las diferentes áreas de las matemáticas en la educación primaria, que estén acordes con las nuevas tendencias internacionales, con los requerimientos de nuestro sistema educativo y que permitan rescatar el potencial con que el estudiantado llega a la escuela; sin embargo, para poder determinar dichas estrategias, es necesario conocer la percepción que tiene el personal docente en cuanto al proceso de enseñanza y aprendizaje de las matemáticas en las aula de primaria; por ello, este estudio particular reporta la percepción que un grupo de docentes de primaria en ejercicio, tiene sobre las matemáticas y su enseñanza, en aspectos como creencias, resolución de problemas, preparación universitaria, capacitaciones, entre otras.

\section{Tipo de investigación}

La investigación realizada para generar el presente documento se puede catalogar de tipo cuantitativa, no experimental descriptiva, pues, de acuerdo con Hernández, Fernández y Baptista (2006) y Cohen y Manion (1990), se describen hechos que sucedieron y el estudio busca generar las interpretaciones correspondientes a esos hechos.

\section{Informantes}

Debido a que el estudio está dirigido a los primeros dos ciclos de la Educación General Básica del país (que incluye los primeros seis años de la educación formal), el profesorado de primaria se convierte en el principal informante de la investigación. La cantidad total de educadores de primaria del país superan los doce mil; no obstante, por las características de la investigación se consideró pertinente tomar una muestra de docentes de primaria de diferentes regiones educativas del país (la muestra es a conveniencia, no es probabilística, por lo que no se puede inferir los resultados a la población de docentes), en total se logró encuestar a 87 personas.

Por la complejidad que podía implicar la recolección de esta información y las características de la investigación, se decidió aprovechar algunos procesos de capacitación que realizó el MEP durante el 2013 para educadores de primaria. Para ello se contó con la colaboración de uno de los asesores nacionales de matemáticas, quien aplicó el cuestionario a docentes que se estaban capacitando. 
ISSN Electrónico: 2215-3470

DOI: http://dx.doi.org/10.15359/ru.30-1.3
UNICIENCIA Vol. 30, No. 1, pp. 31-55. Enero-Junio, 2016. URL: www.revistas.una.ac.cr/uniciencia Email: revistauniciencia@una.cr

\section{Recolección de datos}

La información se recabó por medio de un cuestionario de 66 preguntas, donde las primeras nueve trataban temas generales, luego 57 proposiciones, 45 dirigidas a determinar la percepción docente por las matemáticas y su enseñanza, seis dirigidas a identificar la formación inicial recibida y, por último, seis dirigidas a determinar la percepción sobre los procesos de capacitación y formación recibidos. Se utilizó la escala tipo Likert para estas preguntas, las respuestas se clasificaron por medio de una escala de actitudes, compuesta por cinco categorías, tal como se muestra a continuación:

Tabla 1

Significado de las puntuaciones en la escala de actitudes

\begin{tabular}{lccccc}
\hline & \multicolumn{5}{c}{ Puntuación de la escala } \\
\hline Percepción por & $\mathbf{5}$ & $\mathbf{4}$ & $\mathbf{3}$ & $\mathbf{2}$ & $\mathbf{1}$ \\
\hline Las matemáticas y su enseñanza & $\begin{array}{c}\text { Muy de } \\
\text { acuerdo }\end{array}$ & De acuerdo & Indiferente & En desacuerdo & $\begin{array}{c}\text { Muy en } \\
\text { desacuerdo }\end{array}$ \\
\hline La formación inicial recibida & $\begin{array}{c}\text { Muy } \\
\text { satisfecho }\end{array}$ & Satisfecho & Indiferente & Insatisfecho & $\begin{array}{c}\text { Muy } \\
\text { insatisfecho }\end{array}$ \\
\hline Los procesos de capacitación & $\begin{array}{c}\text { Muy de } \\
\text { acuerdo }\end{array}$ & De acuerdo & Indiferente & En desacuerdo & $\begin{array}{c}\text { Muy en } \\
\text { desacuerdo }\end{array}$ \\
\hline
\end{tabular}

Para resumir la información generada por la aplicación de las preguntas se utilizan algunas medidas estadísticas. Por medio de la media, la mediana y la moda, se determina la percepción, en el sentido de que si estas medidas toman un valor bajo entre 1 y 2,5 se obtiene una opinión negativa, si el valor está entre 2,5 y 3,5 la opinión se puede catalogar como indiferente y si supera a 3,5 se considera una opinión positiva. Por otro lado, se incluye también la desviación estándar, para medir la variabilidad de las respuestas.

Finalmente, debido a que se trabaja con una escala de aptitudes, se utiliza el coeficiente Alpha de Cronbach para determinar la confiabilidad de las preguntas incluidas en el cuestionario.

\section{Estrategias de análisis}

Por medio de la información que proporcione el personal docente, se busca conocer las actitudes y creencias sobre los temas antes mencionados. Para la organización y sistematización de los datos recabados se empleó el software estadístico SPSS (Statistical Package for the Social Sciences).

Para facilitar el análisis de la información, las 45 preguntas vinculadas con la percepción de docentes por las matemáticas y su enseñanza, se subdividieron en seis categorías:

- Creencias personales del personal docente: se incluyen preguntas que permiten analizar directamente los sentimientos personales en relación con las matemáticas.

- Las matemáticas como área disciplinar: se incluyen preguntas por medio de las cuales se pretende identificar la visión del personal docente de las matemáticas no como materia a enseñar sino como área científica; pero dentro de un marco relativamente simple.

- La enseñanza de las matemática por medio de reglas y procedimientos: dentro del marco de los procesos educativos tradicionales, la enseñanza de las matemáticas se ha 
fundamentado en generar un aprendizaje de procedimientos mecánicos para resolver ejercicios matemáticos descontextualizados. Por esta razón, es importante determinar la opinión de docentes sobre este tema.

- Papel de las aplicaciones de las matemáticas: en el marco de la nueva propuesta educativa, se propone que el estudiantado valore la utilidad de las matemáticas para enfrentar problemas de la cotidianidad. Por esta razón, la práctica docente debe involucrar directamente las aplicaciones de la disciplina en diferentes contextos.

- La resolución de problemas en la enseñanza de las matemáticas: como se ha venido indicando, los programas de Matemáticas del MEP establecen la resolución de problemas como la estrategia metodológica y didáctica que debe regir los procesos de enseñanza de la disciplina, por lo que se requiere conocer la opinión de docentes sobre este punto.

- Las creencias del estudiantado en el proceso educativo: se ha discutido ampliamente la importancia de tomar en cuenta las creencias estudiantiles. Tal como lo indican Chaves, Castillo y Gamboa (2008), la sociedad costarricense genera en el estudiantado una serie de prejuicios que afectan directamente el aprendizaje de las matemáticas, por lo que quien educa debe tomar en cuenta dichas creencias para favorecer una enseñanza significativa. En el documento se investigan las creencias de estudiantes en el proceso educativo desde la perspectiva del personal docente.

\section{Resultados}

En total se recabó información de 87 docentes; solamente tres varones. En relación con la categoría profesional que ostentaban en el MEP al momento de la aplicación de la encuesta, se presenta la siguiente tabla 2:

Tabla 2

Distribución de las categorías profesionales a la que pertenece el personal docente entrevistado

\begin{tabular}{ccc}
\hline Categoría profesional & $\begin{array}{c}\text { Número de } \\
\text { docentes }\end{array}$ & Porcentaje \\
\hline PT3 & 2 & 2,3 \\
PT4 & 1 & 1,1 \\
PT5 & 7 & 8,0 \\
PT6 & 77 & 88,6 \\
Total & $\mathbf{8 7}$ & $\mathbf{1 0 0 , 0}$ \\
\hline
\end{tabular}

Los resultados son relevantes en señalar las altas calificaciones profesionales con que contaba el personal docente entrevistado, esto indica que las opiniones recolectadas, estarán respaldadas académicamente por la formación universitaria recibida. Esto se puede complementar con el conocimiento sobre los años de experiencia en educación primaria. Al respecto la tabla 3 resume la información: 
Tabla 3

Distribución de los años de experiencia de 87 docentes en primer $y$ segundo ciclo en la Educación General Básica

\begin{tabular}{lcc}
\hline Años de experiencia & $\begin{array}{c}\text { Número de } \\
\text { docentes }\end{array}$ & Porcentaje \\
\hline Menos de 5 años & 4 & 4,6 \\
De 5 a menos de 10 años & 7 & 8,0 \\
De 10 a menos de 15 años & 24 & 27,6 \\
De 15 a menos de 20 años & 23 & 26,4 \\
De 20 a menos de 25 años & 22 & 25,3 \\
25 años y más & 7 & 8,0 \\
Total & $\mathbf{8 7}$ & $\mathbf{1 0 0 , 0}$ \\
\hline
\end{tabular}

Cerca del $88 \%$ de personal entrevistado contaba con más de 10 años de experiencia como docente de primaria; por lo cual, nuevamente queda en evidencia la importancia de la opinión de estos educadores y educadoras, debido a que corresponderá a la percepción de un grupo con experiencia.

\section{Percepción sobre las matemáticas y su enseñanza}

Como se indicó en la sección anterior, el análisis de este punto se subdividió por categoría que se analizan a continuación.

\section{Percepción sobre sus creencias personales}

En relación con esta área, el cuestionario incluyó 14 preguntas, cuyas respuestas son resumidas en la tabla 4:

\section{Tabla 4}

Percepción de 87 docentes en relación con sus creencias sobre las matemáticas y su enseñanza

\begin{tabular}{lccccc}
\hline \multicolumn{1}{c}{ Ítem } & $\begin{array}{c}\text { No. de } \\
\text { doc. }\end{array}$ & Media & Mediana & Moda & $\begin{array}{c}\text { Desv. } \\
\text { están. }\end{array}$ \\
\hline $\begin{array}{l}\text { Las matemáticas son fundamentales en la formación básica } \\
\text { de la ciudadanía. }\end{array}$ & 81 & 4,3 & 5 & 5 & 1,02 \\
\hline $\begin{array}{l}\text { Disfruto enseñar matemáticas. } \\
\text { Uso las matemáticas para resolver problemas de la vida cotidiana. }\end{array}$ & 81 & 4,5 & 5 & 5 & 0,73 \\
\hline $\begin{array}{l}\text { Disfruto resolver problemas donde están involucrados } \\
\text { conceptos de matemáticas. }\end{array}$ & 80 & 3,9 & 4 & 5 & 1,15 \\
\hline Encuentro interesante el mundo de las matemáticas. & 82 & 4,2 & 4 & 5 & 0,99 \\
\hline $\begin{array}{l}\text { Los problemas de matemáticas me resultan fáciles. } \\
\text { Se deberían eliminar muchos conceptos matemáticos del } \\
\text { currículo de primaria. }\end{array}$ & 80 & 3,7 & 4 & 4 & 1,05 \\
\hline $\begin{array}{l}\text { Las mejores estrategias para favorecer el proceso de } \\
\text { enseñanza y aprendizaje de las matemáticas se orientan } \\
\text { hacia el trabajo individual del estudiante. }\end{array}$ & 82 & 2,4 & 3,5 & 4 & 1,28 \\
\hline
\end{tabular}


UNICIENCIA Vol. 30, No. 1, pp. 31-55. Enero-Junio, 2016.

\begin{tabular}{|c|c|c|c|c|c|}
\hline $\begin{array}{l}\text { Las matemáticas solamente son útiles para la gente de } \\
\text { ciencias y las ingenierías. }\end{array}$ & 81 & 1,6 & 1 & 1 & 0,96 \\
\hline $\begin{array}{l}\text { En I y II Ciclos de la Educación General Básica no se } \\
\text { debería enseñar matemáticas. }\end{array}$ & 80 & 1,2 & 1 & 1 & 0,57 \\
\hline $\begin{array}{l}\text { Me resulta difícil resolver algunos problemas donde se } \\
\text { deben utilizar las matemáticas. }\end{array}$ & 81 & 2,7 & 3 & 4 & 1,28 \\
\hline Me siento intimidado(a) ante problemas de matemáticas. & 80 & 2,2 & 2 & 1 & 1,23 \\
\hline $\begin{array}{l}\text { Para mantener la disciplina y el orden en las lecciones de } \\
\text { matemáticas, es preferible realizar trabajos individuales y } \\
\text { no grupales. }\end{array}$ & 86 & 2,1 & 2 & 1 & 1,19 \\
\hline $\begin{array}{l}\text { La facilidad para comprender las matemáticas es algo con lo } \\
\text { que se nace o no se nace. }\end{array}$ & 81 & 2,1 & 2 & 2 & 1,16 \\
\hline
\end{tabular}

Las respuestas que se generaron son positivas respecto a lo esperado en relación con la propuesta curricular. Los resultados de los diferentes ítems son congruentes al evidenciar gran motivación del personal docente por las matemáticas y su rol en la sociedad. Se presenta una pequeña contradicción en cuanto a la percepción sobre la dificultad en la resolución de problemas matemáticos, debido a que se incluyeron ítems similares en diferentes partes del cuestionario (uno redactado en forma positiva y otro en forma negativa), y se dieron algunas diferencias en los resultados tal como lo muestran las medidas estadísticas. A pesar de los resultados positivos, debe preocupar que, según los datos estadísticos, existen, en este grupo, varios educadores que tienen una percepción contraria a la mayoría, y en contradicción con los planteamientos de los programas de estudio.

\section{Las matemáticas como área disciplinar}

Se incluyeron siete preguntas vinculadas a esta área, la tabla 6 resume los hallazgos.

Tabla 6

Percepción de 87 docentes respecto a las matemáticas

\begin{tabular}{|c|c|c|c|c|c|}
\hline Tema & $\begin{array}{c}\text { No. de } \\
\text { docentes }\end{array}$ & Media & Mediana & Moda & $\begin{array}{l}\text { Desv. } \\
\text { están. }\end{array}$ \\
\hline $\begin{array}{l}\text { El conocimiento matemático no es un descubrimiento } \\
\text { sino una construcción. }\end{array}$ & 83 & 4,4 & 5 & 5 & 0,90 \\
\hline $\begin{array}{l}\text { Los conceptos matemáticos son abstractos, únicamente } \\
\text { pueden ser percibidos y comprendidos por algunas personas. }\end{array}$ & 86 & 2,2 & 2 & 1 & 1,23 \\
\hline $\begin{array}{l}\text { Las matemáticas contribuyen con el desarrollo de cierto } \\
\text { tipo de construcciones mentales. }\end{array}$ & 87 & 4,3 & 4 & 5 & 0,86 \\
\hline $\begin{array}{l}\text { Las matemáticas son un descubrimiento histórico, } \\
\text { universal e invariable. }\end{array}$ & 86 & 3,0 & 3 & 4 & 1,18 \\
\hline $\begin{array}{l}\text { Las matemáticas son verdades absolutas e } \\
\text { inmodificables. }\end{array}$ & 81 & 2,2 & 2 & 2 & 1,16 \\
\hline $\begin{array}{l}\text { Las matemáticas están bien definidas; no están abiertas } \\
\text { a cuestionamientos, argumentos o interpretaciones } \\
\text { personales. }\end{array}$ & 82 & 2,2 & 2 & 1 & 1,16 \\
\hline $\begin{array}{l}\text { Los conocimientos matemáticos no se pueden construir, } \\
\text { ya existen y por lo tanto hay que memorizarlos. }\end{array}$ & 82 & 1,9 & 2 & 2 & 1,02 \\
\hline
\end{tabular}


ISSN Electrónico: 2215-3470

DOI: http://dx.doi.org/10.15359/ru.30-1.3
UNICIENCIA Vol. 30, No. 1, pp. 31-55. Enero-Junio, 2016. URL: www.revistas.una.ac.cr/uniciencia Email: revistauniciencia@una.cr

Anteriormente se evidenció una percepción positiva sobre el rol de las matemáticas dentro de la sociedad; no obstante, los datos de la tabla 6 indican que visualizan la disciplina como un proceso constructivo que está sujeto a argumentación y cuestionamientos. El hecho de que se visualicen las matemáticas como una construcción humana es un importante paso para favorecer una enseñanza más apegada a los intereses de los nuevos programas.

\section{La enseñanza de las matemáticas por medio de reglas y procedimientos}

En el estudio de Edwin Chaves, Castillo, Fonseca, Eduardo Chaves y Loría (2011) para el Estado de la Educación, reflejaron que la enseñanza de las matemáticas en el ámbito de la secundaria en el país se ha regido por estrategias de enseñanza fundamentadas en la memorización de mecanismos y procedimientos dirigidos a la resolución de ejercicios prácticos, carentes de aplicaciones a contextos reales. Por esta razón, se consideró pertinente conocer la opinión de docentes de primaria en relación con el tema. La tabla 7 resume los resultados.

Tabla 7

Percepción de 87 docentes en relación con la necesidad de fundamentar la enseñanza de las matemáticas en reglas y procedimientos

\begin{tabular}{lccccc}
\hline Tema & $\begin{array}{c}\text { No.de } \\
\text { docentes }\end{array}$ & Media & Mediana & Moda & $\begin{array}{c}\text { Desv. } \\
\text { están. }\end{array}$ \\
\hline $\begin{array}{l}\text { Las matemáticas que se enseñan en primaria se } \\
\text { presentan como reglas y procedimientos que el } \\
\text { estudiantado debe reproducir. }\end{array}$ & 84 & 3,7 & 4 & 5 & 1,31 \\
\hline $\begin{array}{l}\text { Para una mejor comprensión de las matemáticas es } \\
\text { preferible centrarse en los conceptos y procedimientos, y } \\
\text { dejar para más adelante sus aplicaciones a otras áreas. }\end{array}$ & 86 & 2,2 & 2 & 2 & 0,99 \\
\hline $\begin{array}{l}\text { Los errores que comete el estudiantado durante las } \\
\text { evaluaciones deben ser objeto de análisis. }\end{array}$ & 83 & 4,3 & 5 & 5 & 0,97 \\
\hline $\begin{array}{l}\text { Las matemáticas implican, principalmente, la aplicación } \\
\text { de reglas y procedimientos para resolver ejercicios. }\end{array}$ & 79 & 2,9 & 3 & 2 & 1,33 \\
\hline $\begin{array}{l}\text { El dominio de las matemáticas se caracteriza por la } \\
\text { habilidad de realizar cálculos aritméticos rápidamente. }\end{array}$ & 80 & 2,4 & 2 & 2 & 1,17 \\
\hline $\begin{array}{l}\text { Las matemáticas es una colección de definiciones y reglas } \\
\text { que hay que memorizar. }\end{array}$ & 82 & 2,0 & 2 & 2 & 1,05 \\
\hline $\begin{array}{l}\text { Las matemáticas implican, principalmente, } \\
\text { memorización de conceptos. }\end{array}$ & 81 & 1,9 & 2 & 1 & 1,09 \\
\hline $\begin{array}{l}\text { El objetivo fundamental de la evaluación en matemáticas } \\
\text { consiste en otorgar una nota que sea congruente con el } \\
\text { desempeño de cada estudiante. }\end{array}$ & 86 & 2,6 & 2,5 & 1 & 1,34 \\
\hline
\end{tabular}

En el resultado del primer ítem, manifiestan claridad al identificar una percepción de que la enseñanza de las matemáticas en primaria constituye el aprendizaje de reglas y procedimientos que deben ser reproducidos; en los restantes ítems se observan algunas inconsistencias respecto al verdadero sentido que debe darse a la acción de aula para favorecer la enseñanza de la disciplina. $\mathrm{Al}$ respecto, se pueden notar algunas concepciones que van en contra con lo planteado en los programas de estudio, donde se pregona que: 
UNICIENCIA Vol. 30, No. 1, pp. 31-55. Enero-Junio, 2016.

ISSN Electrónico: 2215-3470

URL: www.revistas.una.ac.cr/uniciencia

DOI: http://dx.doi.org/10.15359/ru.30-1.3

Email: revistauniciencia@una.cr

Las Matemáticas no son una colección dispersa y desarticulada de conceptosy procedimientos específicos, éstos se integran a partir de ideas y métodos generales cuya construcción y ampliación han sido el resultado de los quehaceres matemáticos. (MEP, 2012, p. 16)

Al referirse a estos métodos tradicionales, en los programas actuales se indica: "Se trata de un estilo de lección que no motiva el interés estudiantil, debilita condiciones centrales para que se potencie la construcción de los aprendizajes" (MEP, 2012, p. 516).

Se incluyeron dos ítems vinculados con la evaluación de los aprendizajes, y se observan contradicciones en las respuestas generadas. Mientras se apoya el hecho de que las evaluaciones tengan un carácter formativo, al mismo tiempo no hay una opinión clara respecto a que el objetivo de las evaluaciones sea simplemente otorgar una nota a los estudiantes.

Por esta razón, la posición de algunas de las personas entrevistadas no es consistente respecto a la posición del MEP y las estrategias de evaluación de aula propuestas.

\section{Papel de las aplicaciones de las matemáticas}

Los Programas de Matemáticas vigentes postulan las aplicaciones de la disciplina como un pilar fundamental sobre el que se sustenta la resolución de problemas. Al respecto se indica:

En este currículo se enfatizará el trabajo con problemas asociados a los entornos reales, físicos, sociales y culturales, o que puedan ser imaginados de esa manera. Se asume que usar este tipo de problemas es una poderosa fuente para la construcción de aprendizajes en las Matemáticas. Al colocarse en contextos reales, el planteo y resolución de problemas conlleva directamente a la identificación, uso y construcción de modelos matemáticos. (MEP, 2012, p. 14)

En relación con el uso de aplicaciones para la enseñanza de las matemáticas, se plantearon, en el cuestionario, seis preguntas, cuyos resultados se presentan en la tabla 8 .

Tabla 8

Percepción de 87 docentes en relación con el papel de las aplicaciones de las matemáticas en el proceso de enseñanza

\begin{tabular}{|c|c|c|c|c|c|}
\hline Tema & $\begin{array}{l}\text { No. de } \\
\text { docentes }\end{array}$ & Media & Mediana & Moda & $\begin{array}{l}\text { Desv. } \\
\text { están. }\end{array}$ \\
\hline $\begin{array}{l}\text { En la enseñanza de las matemáticas en primaria no es necesario } \\
\text { profundizar en representaciones o modelizaciones. }\end{array}$ & 85 & 2,3 & 2 & 1 & 1,21 \\
\hline $\begin{array}{l}\text { Para comprender mejor un concepto matemático es necesario } \\
\text { generar aplicaciones en contextos reales. }\end{array}$ & 85 & 4,53 & 5 & 5 & 0,7 \\
\hline $\begin{array}{l}\text { En la enseñanza de las matemáticas hay que priorizar la contextualización } \\
\text { de experiencias por encima de procedimientos y símbolos. }\end{array}$ & 85 & 3,9 & 4 & 5 & 1,14 \\
\hline $\begin{array}{l}\text { Las experiencias concretas y aplicaciones son importantes para la } \\
\text { comprensión de las matemáticas. }\end{array}$ & 85 & 4,5 & 5 & 5 & 0,77 \\
\hline $\begin{array}{l}\text { Ante la pregunta que realiza regularmente el estudiantado de primaria: ¿para } \\
\text { qué sirven los conceptos matemáticos?, la respuesta debe estar dirigida hacia } \\
\text { su utilidad en estudios posteriores. }\end{array}$ & 85 & 2,9 & 3 & 2 & 1,46 \\
\hline $\begin{array}{l}\text { La enseñanza de las matemáticas debe centrarse en el aprendizaje y } \\
\text { aplicación de los conceptos matemáticos. }\end{array}$ & 84 & 2,9 & 2 & 2 & 1,45 \\
\hline $\begin{array}{l}\text { Los problemas históricos que originaron los conceptos matemáticos, } \\
\text { aunque fueron importantes en su momento, no son necesarios para } \\
\text { tomarlos en cuenta en la enseñanza de la disciplina en primaria. }\end{array}$ & 84 & 2,3 & 2 & 1 & 1,22 \\
\hline
\end{tabular}


Existe mucha inestabilidad en las respuestas vinculadas con esta área, las desviaciones estándar son muy altas para algunos de los ítems. Además, los resultados de las otras medidas estadísticas presentan algunas contradicciones con lo que se debería esperar, según lo que se ha plasmado en los programas oficiales del MEP. Por ejemplo, es contradictorio que en los ítems dos, tres y cuatro se obtenga una opinión favorable hacia la contextualización de experiencias y aplicaciones; pero de las respuestas al primer ítem, se deja entrever ciertas dudas respecto al uso de las representaciones y modelizaciones, las cuales son elementos fundamentales en las aplicaciones matemáticas. Algo similar ocurre con la respuesta al quinto ítem, referido a lo que se debe contestar ante la consulta acerca de ¿para qué sirven los conceptos matemáticos? Se observa que no hay una posición clara sobre la respuesta que se le debe dar a esta pregunta; este tema es tratado muchas veces en los programas del MEP; por ejemplo, seguidamente se plantea una de ellas, donde se indica la importancia que una respuesta adecuada puede tener para que el estudiantado adquiera confianza en la utilidad de la disciplina:

Usar el entorno. El uso de elementos de la zona geográfica en el entorno favorece la percepción de utilidad de las Matemáticas para resolver problemas.

Enfatizar que las Matemáticas sirven para resolver todo tipo de problemas. Desde los primeros años en primaria es esencial que se explique el papel fundamental que las Matemáticas juegan en la resolución de un amplio número de problemas. Se debe visualizar las Matemáticas como algo práctico y sentir que lo que está aprendiendo es muy importante. (MEP, 2012, p. 63)

En general, pareciera que el uso de las aplicaciones para la enseñanza de las matemáticas no ha sido del todo asimilado por este grupo de docentes, en relación con lo plasmado en los programas de estudio.

\section{La resolución de problemas en la enseñanza de las matemáticas}

En cuanto a la resolución de problemas como estrategia didáctica

La resolución de problemas como estrategia pedagógica se subrayará aquí como sustrato de un estilo de acción de aula. Para el aprendizaje de conocimientos dentro de la lección se propone una introducción de los nuevos tópicos que tome en cuenta cuatro pasos o momentos centrales:

(1) propuesta de un problema,

(2) trabajo estudiantil independiente,

(3) discusión interactiva y comunicativa,

(4) clausura o cierre.

Esta secuencia puede realizarse dentro de una lección o una colección de ellas de acuerdo con el tema o el año lectivo. Este estilo se contrapone a aquel que trabaja los tópicos matemáticos en abstracto, ofrece ejemplos y prácticas rutinarias y al final, como apéndice, ejercicios o problemas contextualizados. No se trata de una prescripción a seguirse mecánicamente, pues su diseño y realización depende de las condiciones donde se plantee el aprendizaje. 
UNICIENCIA Vol. 30, No. 1, pp. 31-55. Enero-Junio, 2016.

Email: revistauniciencia@una.cr

Usar problemas se propone como una constante en todas las fases de la acción de aula, incluyendo aquella del reforzamiento, movilización y aplicación de los conocimientos aprendidos. (MEP, 2012, pp.14-15)

Con lo planteado anteriormente, se constata que la resolución de problemas juega un papel relevante en la nueva propuesta educativa. En la tabla 9, se plantea un resumen vinculado con las respuestas dadas a los ítems vinculados con este tema.

Tabla 9

Percepción de 87 docentes respecto a la resolución de problemas en el proceso de enseñanza de las matemáticas

\begin{tabular}{lccccc}
\hline \multicolumn{1}{c}{ Tema } & $\begin{array}{c}\text { No. de } \\
\text { docentes }\end{array}$ & Media & Mediana & Moda & $\begin{array}{c}\text { Desv. } \\
\text { están. }\end{array}$ \\
\hline $\begin{array}{l}\text { Se debe enseñar matemáticas a partir de la resolución } \\
\text { de problemas rutinarios. }\end{array}$ & 86 & 3,9 & 4 & 5 & 1,36 \\
\hline $\begin{array}{l}\text { Es fundamental explicar detalladamente cada concepto } \\
\text { matemático y dar ejemplos antes de permitir que el } \\
\text { estudiantado se enfrente a un problema matemático. }\end{array}$ & 86 & 2,6 & 2 & 2 & 1,38 \\
\hline $\begin{array}{l}\text { Los problemas matemáticos que se le plantean al } \\
\text { estudiantado deben resolverse en poco tiempo para } \\
\text { favorecer el aprendizaje. }\end{array}$ & 86 & 2,3 & 2 & 2 & 1,18 \\
\hline $\begin{array}{l}\text { Los problemas matemáticos deben ser retadores para el } \\
\text { estudiantado y obligarle a generar nuevas ideas. }\end{array}$ & 80 & 4,1 & 4 & 4 & 1,01 \\
\hline $\begin{array}{l}\text { Las matemáticas nos ayudan en la resolución de } \\
\text { problemas cotidianos. }\end{array}$ & 81 & 4,6 & 5 & 5 & 0,79 \\
\hline
\end{tabular}

Aunque las respuestas anteriores reflejan una percepción positiva en relación con la resolución de problemas como estrategia didáctica, por la importancia que se le da a este tema en los nuevos programas, se esperarían datos más contundentes en relación con el tema. Es decir, se esperaría un convencimiento absoluto sobre la importancia de la resolución de problemas como estrategia metodológica para la labor de aula. Sobre todo si se analiza la gran variabilidad en las respuestas que se generan en los dos primeros ítems, lo que refleja la ausencia de una puesta en común con este tema.

\section{Las creencias del estudiantado en el proceso educativo}

Así como se ha manifestado a lo largo del documento la importancia que juegan las actitudes y creencias docentes; no son menos importantes, las creencias con que el estudiantado inicia un curso lectivo. Por esta razón, se plantearon cuatro ítems vinculados con este aspecto. Las respuestas se resumen en la tabla 10. 
ISSN Electrónico: 2215-3470

DOI: http://dx.doi.org/10.15359/ru.30-1.3
UNICIENCIA Vol. 30, No. 1, pp. 31-55. Enero-Junio, 2016.

URL: www.revistas.una.ac.cr/uniciencia Email: revistauniciencia@una.cr

\section{Tabla 10}

Percepción de 87 docentes respecto al papel de las creencias de estudiantes en el proceso de enseñanza de las matemáticas

\begin{tabular}{lccccc}
\hline Tema & $\begin{array}{c}\text { No. de } \\
\text { docentes }\end{array}$ & Media & Mediana & Moda & $\begin{array}{c}\text { Desv. } \\
\text { están. }\end{array}$ \\
\hline $\begin{array}{l}\text { La percepción que tiene el estudiantado sobre el grado } \\
\text { de dificultad de las matemáticas, debe ser tomada en } \\
\text { cuenta para elaborar estrategias que permitan cambiar } \\
\text { esas ideas. }\end{array}$ & 86 & 4,4 & 5 & 5 & 0,94 \\
\hline $\begin{array}{l}\text { En el proceso educativo no se deben tomar en cuenta } \\
\text { las creencias matemáticas que tiene el estudiantado, } \\
\text { pues pueden ser equivocadas. }\end{array}$ & 81 & 2,5 & 2 & 2 & 1,09 \\
$\begin{array}{l}\text { Es importante provocar que el estudiantado ajuste sus } \\
\text { creencias de acuerdo con los contenidos matemáticos } \\
\text { que se desarrollan. }\end{array}$ & 82 & 3,2 & 3 & 4 & 1,15 \\
\hline $\begin{array}{l}\text { Es natural que la complejidad de las matemáticas } \\
\text { y su aprendizaje provoquen inseguridad entre el } \\
\text { estudiantado. }\end{array}$ & 82 & 3,4 & 4 & 4 & 1,38 \\
\hline
\end{tabular}

Los resultados del primer ítem revelan que el personal docente opina que las percepciones estudiantiles sobre el grado de dificultad de las matemáticas deben ser tomadas en cuenta para planificar las lecciones; los datos relacionados con los resultados de los otros ítems dejan entrever cierta contradicción, debido a que en ellos se plantea una posición neutra, lo que demuestra que no se tiene plena claridad respecto al tema. En especial preocupan los resultados del último ítem, donde existe una ligera tendencia a suponer como natural que las matemáticas y su aprendizaje generen inseguridad. El tema es preocupante, debido a que la percepción de que las matemáticas y su enseñanza son complejas puede plantear una barrera para potenciar una enseñanza consistente con los programas de estudio. Respecto a este tema, en los programas vigentes se indica:

Lo primero, es un cambio de visión, de estilo: los programas quieren contribuir a romper el mito de que las Matemáticas son áridas, feas, imposiblemente difíciles y algo de lo que los estudiantes tienen que sentir miedo. Esta visión de las Matemáticas como "el coco" ha sido tradicional en Costa Rica y en casi todo el mundo, y es uno de los prejuicios que hay que superar para lograr un cambio significativo en nuestra capacidad de enseñar y aprender Matemáticas. Se trata de familiarizar al estudiante con las [sic] Matemática, de hacérsela cercana, agradable, emocionante. (MEP, 2012, p. 10)

Lo que indica que en los programas de estudio vigente se pretende que en las aulas se supere el prejuicio histórico que ha afectado tanto la enseñanza de esta disciplina. En cuanto a la importancia de las creencias de estudiantes, se plantea en los programas de estudio como un eje transversal:

A su vez, para lograr una actitud distinta de los estudiantes hacia las matemáticas, desarrollando actitudes y creencias positivas sobre las matemáticas, desarrollando el gusto por las matemáticas, se plantean cinco actitudes a desarrollar: perseverancia; confianza en la 
UNICIENCIA Vol. 30, No. 1, pp. 31-55. Enero-Junio, 2016.

utilidad de las Matemáticas; participación activa y colaborativa; autoestima en relación con el dominio de las Matemáticas; respeto, aprecio y disfrute de las Matemáticas. (MEP, 2012, p.11)

Más adelante se indica:

Se asume explícitamente el fortalecimiento de actitudes, creencias y valores positivos sobre las Matemáticas como eje disciplinar transversal, lo que no sólo contribuye al desenvolvimiento de la personalidad individual de quien participa en la acción educativa, sino que ensancha el espacio de los valores y las actitudes en general, como la solidaridad y la acción cooperativa. (MEP, 2012, p.20)

Lo anterior deja en evidencia la importancia que se atribuye a este tema en la nueva propuesta curricular, por lo que la información suministrada por los educadores y educadoras deja patente que el tema debe ser motivo de análisis por parte de las autoridades magisteriales.

Para finalizar esta sección, es importante valorar el nivel de confiabilidad de las preguntas incluidas en el cuestionario relacionadas con este tema. En este sentido, tal como se indicó antes, se utiliza el coeficiente Alpha de Cronbach para establecer dicha confiabilidad. El valor de este coeficiente para los 45 ítems fue de 0,80; el cual se considera muy aceptable en el análisis de las escalas de actitudes.

\section{Percepción sobre formación universitaria recibida}

Ha sido discutida ampliamente la importancia que juega la labor docente para que la implementación de los nuevos programas sea exitoso; en este sentido, debido a que el mismo estudio ha demostrado que las 87 personas docentes han sido formados en diferentes programas académicos de distintas universidades; es necesario, entonces, que, con base en la experiencia docente alcanzada, el profesorado se manifieste respecto de algunos puntos vinculados con su formación universitaria base. La tabla 11 resume dicha información.

Tabla 11

Percepción de 87 docentes respecto a la formación universitaria recibida para enseñar matemáticas

\begin{tabular}{|c|c|c|c|c|c|}
\hline $\begin{array}{l}\text { Grado de satisfacción respecto por la formación } \\
\text { recibida }\end{array}$ & $\begin{array}{c}\text { No. de } \\
\text { docentes }\end{array}$ & Media & Mediana & Moda & $\begin{array}{l}\text { Desv. } \\
\text { están. }\end{array}$ \\
\hline En aspectos teóricos de las matemáticas. & 86 & 2,9 & 2 & 2 & 1,12 \\
\hline En estrategias didácticas para enseñar matemáticas. & 86 & 2,6 & 2 & 2 & 1,14 \\
\hline En estrategias evaluativas en educación matemática. & 85 & 2,5 & 2 & 2 & 1,07 \\
\hline En el conocimiento de los programas oficiales del MEP. & 86 & 2,7 & 3 & 4 & 1,20 \\
\hline $\begin{array}{l}\text { En la relación entre el plan de estudios de la carrera y } \\
\text { los programas de estudios del MEP. }\end{array}$ & 87 & 2,5 & 2 & 2 & 1,12 \\
\hline Para impartir lecciones de matemáticas en primaria. & 85 & 3,0 & 3 & 2 & 1,25 \\
\hline
\end{tabular}

Las medidas estadísticas para estos ítems denotan una clara tendencia a concebir como regular o deficiente la formación universitaria recibida para laborar como profesor o profesora de primaria en el área de matemáticas. Este es un aspecto que requiere de especial atención, 
debido a que, si el personal docente no está satisfecho con la formación universitaria recibida puede estar afectando el proceso de enseñanza y aprendizaje de las matemáticas en las aulas de primaria; además, la mayoría son altamente calificados en el MEP, con títulos que les respalda y aun así no están del todo no evidencian satisfacción con su formación.

Se debe resaltar en las respuestas vinculadas con este tema que existió mucha variabilidad en las respuestas dadas, prueba de ello corresponde a los valores de la desviación estándar. Por otro lado, en cuanto a la confiabilidad del instrumento para este tema, el coeficiente Alpha de Cronbach alcanzó un valor de 0,86 ; lo que demuestra una alta confiabilidad con las preguntas incluidas en el cuestionario.

El descontento manifestado con la formación profesional recibida puede relacionarse, con lo que se apuntaba anteriormente, respecto a que en los programas de formación de docentes de I y II Ciclo de la Educación General Básica tienen muy pocos cursos relacionados con matemáticas y su didáctica.

\section{Percepción por los procesos de actualización y capacitación recibidos}

En el punto anterior quedó en evidencia que, para el personal docente entrevistado, no existe satisfacción por la formación universitaria recibida en el campo de la enseñanza de las matemáticas. Por esta razón, para llenar los vacíos que puede haber dejado la formación universitaria, los procesos de capacitación y actualización se convierten en una vía alternativa para suplir las aparentes deficiencias. En este sentido, se consideró pertinente incluir varias preguntas para determinar la percepción de este grupo en relación con estos procesos. En la tabla 12, se muestran los resultados obtenidos.

Tabla 12

Percepción de 87 docentes respecto a los procesos de capacitación y actualización en el campo de la enseñanza de las matemáticas

\begin{tabular}{lccccc}
\hline $\begin{array}{l}\text { Grado de satisfacción respecto a las capacitaciones y } \\
\text { actualizaciones }\end{array}$ & $\begin{array}{c}\text { No. de } \\
\text { docentes }\end{array}$ & Media & Mediana & Moda & $\begin{array}{c}\text { Desv. } \\
\text { están. }\end{array}$ \\
\hline $\begin{array}{l}\text { Se han desarrollado en un número que ha estado en } \\
\text { concordancia con sus necesidades. }\end{array}$ & 85 & 2,4 & 2 & 2 & 1,22 \\
\hline $\begin{array}{l}\text { Le han permitido fortalecer sus conocimientos teóricos } \\
\text { matemáticos. }\end{array}$ & 85 & 2,9 & 3 & 4 & 1,23 \\
\hline $\begin{array}{l}\text { Le han permitido fortalecer sus conocimientos sobre } \\
\text { estrategias didácticas para la enseñanza de las matemáticas. }\end{array}$ & 85 & 2,9 & 3 & 4 & 1,25 \\
\hline $\begin{array}{l}\text { Le han permitido fortalecer los procesos de planificación } \\
\text { y evaluación para las lecciones de matemáticas. }\end{array}$ & 83 & 2,6 & 2 & 2 & 1,21 \\
\hline $\begin{array}{l}\text { Le han permitido conocer las nuevas tendencias sobre } \\
\text { la enseñanza de las matemáticas. }\end{array}$ & 85 & 3,1 & 4 & 4 & 1,26 \\
\hline Le han sensibilizado sobre el rol de los programas de estudio. & 85 & 3,3 & 4 & 4 & 1,29 \\
\hline
\end{tabular}

Desafortunadamente, tampoco en este rubro existe una buena percepción del personal docente. Por el contrario, con excepción de los últimos dos ítems que tienen datos medianamente aceptables, y que posiblemente se relacionan con los programas de capacitación vinculados con los nuevos programas de estudio y que surgieron recientemente al momento en que se aplicó la encuesta; los demás ítems tienen resultados no muy positivos y que demuestran que no hay 
gran satisfacción por lo que han sido estos procesos tradicionalmente. En los programas de matemáticas se indica:

Las universidades formadoras de docentes no le han dado el lugar que debería tener en sus planes de formación inicial. Eso obligaría a modificaciones importantes en esos planes (cf. Ruiz, Barrantes y Gamboa, 2009, cf. Araya y Sequeira, 2008). También, y esto es central: plantea desencadenar amplios procesos de capacitación en servicio, una auténtica formación continua. (MEP, 2012, p. 513)

Por ello, este es un tema central que debe ser tomado en cuenta por las autoridades del MEP y las mismas universidades, que no pueden evadir la responsabilidad de cumplir sus objetivos: se deben crear las condiciones que garanticen el éxito en la implementación de los programas.

Para finalizar, el coeficiente Alpha de Cronbach para este último bloque de preguntas alcanzó un valor de 0,92 ; por lo que queda garantizada una vez más la confiabilidad del instrumento utilizado para determinar la opinión de los docentes.

\section{Conclusiones}

Desde la perspectiva de las nuevas corrientes pedagógicas, ha quedado claramente evidenciada la importancia de incorporar los aspectos afectivos dentro de los procesos educativos. El presente documento constituye un aporte en ese sentido. Sin embargo, se debe hacer notar que lo que se ha pretendido es identificar la percepción de un grupo de docentes de primaria en relación con las matemáticas y su enseñanza desde la perspectiva de los Programas de Matemáticas del MEP.

En términos generales, ha quedado demostrado que en el personal docente entrevistado se tiene una percepción positiva en cuanto a las matemáticas y su enseñanza, además se concibe la disciplina como una construcción humana a través de la historia. También se valora positivamente el empleo de la resolución de problemas como una estrategia metodológica para la enseñanza de la disciplina.

Estos elementos resultan muy positivos para favorecer un adecuado proceso de enseñanza de las matemáticas en el ámbito de la primaria. No obstante, algunos de los resultados obtenidos dejan entrever otros aspectos que deben ser tomados en cuenta, pues constituyen serias barreras para lograr dicho propósito. Seguidamente se plantea un análisis de cada uno de ellos.

Se puede observar, que en parte del grupo de docentes que se entrevistaron se les hace difícil resolver problemas cuya solución requiere del uso de las matemáticas. Este elemento debe ser muy bien valorado, pues en vez de una debilidad se podría considerar como una oportunidad para evidenciar que los problemas que se deben plantear en la primaria deben ser retadores y, por ende, para la búsqueda de una solución se requiere de un proceso de reflexión, análisis y razonamiento lógico. En este sentido, resulta natural que la búsqueda de soluciones resulte difícil, lo importante es que sea viable encontrar soluciones por medio de herramientas matemáticas en congruencia con el nivel educativo en que dicho problema se esté planteando.

Un tema de mayor preocupación consiste en la percepción, de un importante número de docentes, a favor de eliminar algunos temas de matemática en primaria. Desafortunadamente, no se tienen los argumentos que fundamentan esta percepción, por lo que únicamente se pueden plantear algunas hipótesis al respecto. Esta opinión negativa puede obedecer a que no se aprecia la utilidad de dichos temas en la formación de la ciudadanía o, por el contrario, a que sean 
conceptos complejos sobre los cuales no se tiene un pleno dominio, por cuanto se podrían estar sintiendo intimidación al tener que enseñar conceptos que no se conocen, por ello se prefiere que se eliminen del currículo.

Aunque los educadores y educadoras participantes en el estudio percibieron el análisis de las creencias estudiantiles al momento de planear las actividades de aula, se percibe también que las matemáticas pueden provocar inseguridad en sus estudiantes. Este análisis es consecuente con su creencia particular sobre la concepción de las matemáticas como una disciplina de difícil comprensión. Al vincular estos elementos, se advierte la preocupación de que dicha concepción podría generar barreras psicológicas al momento de planificar el proceso educativo y subestimar el potencial del estudiantado para enfrentar la resolución de problemas por medio de la aplicación de estrategias matemáticas.

Uno de los aspectos que resulta de mayor preocupación consiste en la percepción sobre la formación universitaria para fungir como docentes de primaria, especialmente para la enseñanza de las matemáticas. Específicamente, los encuestados sienten poca satisfección en relación con aspectos teóricos, estrategias de enseñanza y de evaluación; no sienten que la formación recibida durante sus años universitarios estuviera en estrecha relación con la labor profesional que deberían realizar en las aulas de primaria. Este debe ser un tema de análisis de las diferentes instancias que forman educadores de primaria en el país; pero también del MEP al momento de su contratación, pues debería existir algún proceso que valide sus competencias en función de los requerimientos de los programas de estudio. Esto cobra especial relevancia, si se considera que el análisis realizado en el presente estudio, ha dejado en evidencia la multiplicidad de universidades y programas destinados a la formación de docentes de I y II Ciclos de la Educación General Básica, los cuales tienen diferentes énfasis y se atiende la formación en educación matemática de formas muy diversas.

Aunado a lo anterior, si no se recibe una formación universitaria coherente con los programas de estudio del MEP para la enseñanza de las matemáticas, los procesos de capacitación y actualización se constituyen en una herramienta de primordial importancia para complementar la formación. Sin embargo, la percepción del personal docente entrevistado en relación con los procesos que han recibido, no es muy positiva y se presenta mucha variación en las opiniones. Consideran que no han sido suficientes los procesos y que no se han abordado todas las temáticas requeridas como la evaluación en el aula. Esto muestra una relación directa con lo expuesto en el Estado de la Educación 4 (2013) cuando señala que respecto al seguimiento posterior a la capacitación y sobre el uso que se da en el aula a lo aprendido en los cursos:

Llama la atención el poco acompañamiento que reciben los docentes por parte de quienes imparten la capacitación, hecho que constituye una de las principales falencias del actual modelo de formación profesional. En cuanto a la aplicación en el aula de los conocimientos adquiridos, se detectó la necesidad de ampliar la oferta con cursos que orienten sobre las nuevas formas en que hoy aprenden los estudiantes y las diversas estrategias que los docentes pueden utilizar en el aula para mejorar su interacción con los alumnos. (p. 166)

No obstante, cabe mencionar que a diferencia de procesos anteriores en los que se han instaurado nuevos programas de matemáticas, sin el acompañamiento oportuno y adecuado, esta nueva propuesta curricular ha venido acompañada de una serie de procesos de sensibilización, 
actualización y capacitación para apoyar al personal docente en su tarea. Desafortunadamente, al momento de la aplicación de esta encuesta, dichos procesos recién iniciaban, por lo que sus resultados no fueron consignados plenamente dentro de los hallazgos de ella. Sin embargo, se debe destacar que, a pesar de lo anterior, una buena cantidad manifestó satisfacción con las capacitaciones relacionadas con el conocimiento e implementación de los nuevos programas.

Por todo lo anterior, se ha reflejado en el documento que, en relación con los tópicos analizados en el presente estudio, para fortalecer la enseñanza de las matemáticas en primaria, las diferentes instancias y personas involucradas en el área: autoridades del MEP y asesorías de matemáticas, instancias capacitadoras, responsables de programas formadores de educadores y profesorado de primaria, entre otros, deben reflexionar profundamente para establecer estrategias que permitan encontrar concordancia entre los principios matemáticos que requiere nuestra juventud en primaria, la propuesta curricular y la realidad de aula, de modo que los propósitos básicos que sustentan los planes de estudio puedan ser llevados efectivamente a la práctica.

\section{Referencias}

Alfaro, A., Alpízar, M., Morales, Y., Salas, O. y Ramírez, M. (2013). La formación inicial y continua de docentes de matemáticas en Costa Rica. Cuadernos de Investigación y formación en Educación Matemática, año 8(especial), 131-179. Recuperado de http://revistas.ucr.ac.cr/index.php/cifem/article/ view/12225/11496

Alpízar, M., Barrantes, J. P., Bolaños, H., Céspedes, M., Delgado, E., Freer, D., Padilla, E. y Víquez, M. (2012). Aspectos relevantes sobre la formación docente en I y II ciclos en los temas probabilidad y estadística. Revista Electrónica Educare, 16(2), 113-129. Recuperado de http://www.revistas.una.ac.cr/ index.php/EDUCARE/article/view/3934

Barrantes, J., Bolaños, H., Céspedes, M., Delgado, E., Freer, D., Padilla, E. y Víquez, M. (2010). Estado actual de la enseñanza y aprendizaje de probabilidad y estadística, en I y II Ciclos, en la educación costarricense en las direcciones regionales educativas de Heredia y Pérez Zeledón (Tesis de Licenciatura). Universidad Nacional de Costa Rica. Heredia, Costa Rica.

Bosch, M. y Gascón, J. (2001) . Las prácticas docentes del profesor de matemáticas. (versión preliminar) XIème École d'Été de Didactique desMathématiques. Recuperado de http://www.ugr.es/ jgodino/ siidm/almeria/Practicas_docentes.PDF

Chaves, E., Castillo, M. y Gamboa, R. (2008). Creencias de los estudiantes en los procesos de aprendizaje de las matemáticas. Cuadernos de Investigación y Formación en Educación Matemática, 3(4), 29-44.

Chaves, E., Castillo, M. y Alpízar, M. (2009). Percepción de profesores de matemática sobre la estadística y su enseñanza. Acta Latinoamericana de Matemática Educativa, 22, 1472-1482. Recuperado de www. clame.org.mx/documentos/alme22.pdf

Chaves, E., Castillo, M., Fonseca, J., Chaves, E. y Loría, R. (2011). La enseñanza de las matemáticas en la secundaria costarricense: Entre la realidad y la utopía. En Programa Estado de la Nación en Desarrollo Humano Sustentable, III Informe del Estado de la Educación, CONARE. Recuperado de: http:// www.estadonacion.or.cr/images/stories/informes/educacion_003/docs/ponencias/Chavez_2010_ Matematica.pdf

Cohen, L. y Manion, L. (1990). Métodos de investigación educativa. España: Editorial La Muralla.

Estrada, A. (2002). Análisis de las actitudes y conocimientos estadísticos elementales en la formación del profesorado (Tesis doctoral). Universitat Autònoma de Barcelona, Departament de Didàctica de les Matemàtiques i les Ciències Experimentals, Barcelona. 
ISSN Electrónico: 2215-3470

DOI: http://dx.doi.org/10.15359/ru.30-1.3
UNICIENCIA Vol. 30, No. 1, pp. 31-55. Enero-Junio, 2016. URL: www.revistas.una.ac.cr/uniciencia Email: revistauniciencia@una.cr

Flores, P. (1998). Creencias y concepciones de los futuros profesores sobre las matemáticas, su enseñanza y aprendizaje. Evolución durante las prácticas de enseñanza (Tesis doctoral inédita). Universidad de Granada, Departamento de Didáctica de la Matemática, Granada. Recuperado de http://www.ugr. es/ pflores/textos/aRTICULOS/Investigacion/UNOTessis.pdf

Hernández, R., Fernández, C. y Baptista, P. (2006). Metodología de la investigación (cuarta edición). México: McGraw-Hill Interamericana.

McLeod, B., \& McLeod, S. (2002). Synthesis- Beliefs and Mathematics Education: Implications for learning, teaching, and approach. En G. Leder, E. Pehkonen, \& G.Törner (Eds.), Beliefs: A hidden variable in Mathematics Education?, (pp. 115-123) Kluwer Academic Plublishers. Netherlands.

Ministerio de Educación Pública (MEP). (2012). Programas de Estudio en Matemáticas para la Educación General Básica y el Ciclo Diversificado. San José, Costa Rica: autor.

Pozo J. I. y Scheuer, M. (2006). Nuevas formas de pensar la enseñanza y el aprendizaje. Barcelona, España: Grao. Programa Estado de la Nación. (2013). Cuarto Informe Estado de la Educación. San José, Costa Rica: Autor. Recuperado de http://www.estadonacion.or.cr/files/biblioteca virtual/educacion/004/8-Cap-3.pdf

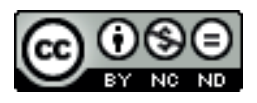

Percepción de los docentes de primaria, en ejercicio, acerca de las Matemáticas y su enseñanza en relación con los programas oficiales del MEP (Edwin Chaves-Esquivel y otros) por Revista Uniciencia se encuentra bajo una Licencia CreativeCommons Atribución-NoComercial-SinDerivadas 3.0 Unported. 